\title{
Light-sheet photonic force optical coherence elastography for high-throughput quantitative 3D micromechanical imaging
}

\section{Yuechuan Lin}

Massachusetts Institute of Technology https://orcid.org/0000-0002-1336-0668

Nichaluk Leartprapun

Cornell University https://orcid.org/0000-0002-3848-9695

Justin Luo

Cornell University

Steven Adie ( $\nabla$ sga42@cornell.edu )

Cornell University

\section{Article}

Keywords: extracellular matrix, LS-pfOCE, quantitative 3D imaging

Posted Date: September 23rd, 2021

DOI: https://doi.org/10.21203/rs.3.rs-900142/v1

License: (9) This work is licensed under a Creative Commons Attribution 4.0 International License. Read Full License

Version of Record: A version of this preprint was published at Nature Communications on June 16th, 2022. See the published version at https://doi.org/10.1038/s41467-022-30995-0. 


\title{
Light-sheet photonic force optical coherence elastography for high-throughput quantitative 3D micromechanical imaging
}

\author{
Yuechuan Lin ${ }^{1,2}$, Nichaluk Leartprapun ${ }^{1,2}$, Justin C. Luo ${ }^{1,2} \&$ Steven G. Adie ${ }^{1, *}$ \\ ${ }^{1}$ Meinig School of Biomedical Engineering, Cornell University, Ithaca, NY 14853, USA. \\ ${ }^{2}$ These authors contribute equally. \\ (Y.L. present affiliation Department of Mechanical Engineering, Massachusetts Institute of Technology, Cambridge, \\ MA 02139, USA; N.L. present affiliation Wellman Center for Photomedicine, Massachusetts General Hospital, \\ Harvard Medical School, Boston, MA 02114, USA.)
}

\begin{abstract}
Microscale mechanical properties of the extracellular matrix (ECM) and dynamic cell-ECM interactions play an important role in pathophysiological processes, including the onset, progression, and attenuation of disease. The ability to quantitatively image cell-mediated micromechanical dynamics of the ECM in physiologically relevant 3D engineered cellular systems can significantly enhance the clinical translational potential of fundamental discoveries in the rapidly growing field of mechanobiology. However, it remains a challenge for current mechanical characterization methods to combine quantitative 3D imaging of ECM mechanics with cellular-scale resolution and dynamic monitoring of cell-mediated changes to pericellular viscoelasticity. Here, we present light-sheet photonic force optical coherence elastography (LS-pfOCE) to address this challenge by leveraging a light-sheet for parallelized, non-invasive, and localized mechanical loading. We demonstrate the capabilities of LS-pfOCE by imaging the micromechanical heterogeneity of fibrous 3D collagen matrices and perform a live-cell study to image micromechanical heterogeneity induced by NIH-3T3 cells seeded in 3D fibrin constructs. We also show that LSpfOCE is able to quantify temporal variations in pericellular viscoelasticity in response to drug-induced altered cellular activity. By providing access to 4D spatiotemporal variations in the micromechanical properties of 3D biopolymer constructs and engineered cellular systems, LS-pfOCE has the potential to drive new discoveries in mechanobiology and contribute to the development of novel biomechanics-based clinical diagnostics and therapies.
\end{abstract}


Mechanical properties of the extracellular matrix (ECM) and biological tissues play an important role in regulating cellular functions ${ }^{1,2}$. The ECM not only provides a physical scaffold for cell adhesion and migration ${ }^{3}$, but the mechanical properties of the ECM also serve as prominent mechanical cues in various pathophysiological processes ${ }^{4}$. Reciprocally, cells can also alter the micromechanical heterogeneity and stiffness of the ECM via cell-mediated matrix deformation and degradation ${ }^{5,6}$. In the rapidly growing field of mechanobiology, importance has been given to the study of these bi-directional cell-ECM biomechanical interactions in physiologically relevant three-dimensional (3D) environments ${ }^{7,8}$. Given the dynamic nature of biological systems, the ability to characterize 3D spatial and temporal variations of ECM mechanical properties at the cellular-scale could be useful for understanding the role of biomechanical cell-ECM interactions in physiological processes such as stem cell differentiation ${ }^{9-11}$, morphogenesis ${ }^{12}$, and wound healing ${ }^{11}$, as well as the onset and/or progression of diseases including cancer ${ }^{13-18}$, muscular dystrophy ${ }^{19}$, and calcific aortic valve diseases ${ }^{20,21}$. Thus, a method for high-throughput 3D quantitative micromechanical imaging of the ECM in engineered cellular systems has the potential to unlock new avenues of research in the field of mechanobiology.

Despite the efforts in the development of a variety of techniques for mechanical characterization of biological tissues and engineered ECM constructs ${ }^{22-34}$, it still remains as a challenge for current techniques to simultaneously support cellular-scale spatially-resolved measurements, practical 3D volumetric acquisition times, and quantitative reconstruction of mechanical properties. Conventional bulk mechanical testing methods such as shear rheometry are unsuitable for spatially-resolved live-cell imaging studies ${ }^{22}$. On the other hand, atomic force microscopy (AFM) - the gold standard for high-resolution mapping of stiffness - is only capable of 2D measurements on the surface of the sample $^{23}$. Emerging optical elastography techniques are under development to fill the gaps between these two extremes. Existing optical coherence elastography (OCE) techniques are most suitable for tissue-level measurements (due to the geometric size of applied mechanical loading) or do not support quantitative reconstruction of viscoelastic properties ${ }^{24-}$ ${ }^{26}$. Brillouin microscopy has demonstrated 3D micromechanical imaging with sub-cellular resolution, but accurate physiological interpretation of the measured $\mathrm{GHz}$ longitudinal modulus in relation to the more conventional shear or Young's modulus remains a challenge ${ }^{27-29}$. Particle-tracking passive microrheology is capable of quantitative measurements in 3D, but it is predominantly applicable in highly compliant (shear modulus <100 Pa) viscositydominant materials ${ }^{30-34}$. Alternatively, optical tweezer-based active microrheology (OT-AMR) can support microrheological measurements in more rigid elasticity-dominant viscoelastic material $\mathrm{s}^{30-34}$. However, the practicality of OT-AMR for volumetric measurements is still limited due to the need to serially align (with $0.1-\mu \mathrm{m}$ precision) the high numerical aperture (NA) trapping and detection beams to individual probe beads that are randomly distributed in 3D space.

We present light-sheet photonic force OCE (LS-pfOCE), an all-optical method based on a novel use of photonic radiation pressure from a light-sheet for alignment-free parallel optical micromanipulation, to address the persisting need for high-throughput quantitative 3D micromechanical characterization in the field of mechanobiology. LSpfOCE can support volumetric imaging with quantitative reconstruction of viscoelasticity in 3D physiologically relevant ECM constructs and engineered cellular systems. We validate and demonstrate LS-pfOCE in polyacrylamide (PAAm) gels, collagen matrices with varying fibre architecture, and live-cell imaging of cell-mediated ECM remodelling in 3D fibrin hydrogels seeded with NIH-3T3 fibroblast cells.

\section{Results \\ Principle of photonic force $\mathrm{OCE}$}

Inspired by the pioneering work of Nobel laureate Arthur Ashkin ${ }^{35}$, we exploited a less conventional mode of optical manipulation, based on radiation pressure from a low-NA beam, to develop photonic force (PF)-OCE for 3D mechanical microscopy ${ }^{36}$. PF-OCE utilizes radiation pressure from a low-NA beam (NA $\leq 0.4$, compared to NA $\geq 1$ in conventional high-NA OTs) to provide "AFM-like" localized mechanical loading that is applied to micron-sized probe beads randomly distributed in 3D space. Leveraging interferometric detection from phase-sensitive optical coherence tomography (OCT), oscillations of these probe beads induced by harmonically modulated radiation pressure can be detected with sub-nanometer displacement sensitivity after compensating for the confounding photothermal (PT) response of the medium. The measured complex mechanical responses of individual probe beads reflect the local viscoelastic properties of the medium in the vicinity of each bead (i.e., bead "microenvironment"). With the extended depth coverage afforded by the use of a low-NA beam, and no prerequisite for precise bead-wise optical alignment 
that restricts the practicality of OT-AMR, PF-OCE is well-suited for volumetric measurements in 3D engineered cell culture systems, with additional benefits of OCT providing label-free, rapid volumetric imaging in scattering media.

Nevertheless, our previous implementation of PF-OCE for 3D mechanical microscopy, based on transverse rasterscanning of a Gaussian forcing beam, resulted in a pulse-train temporal excitation profile on each probe bead instead of the desired continuous sinusoidal waveform (see Supplementary Fig. $2 \mathrm{~b}$ in Ref. ${ }^{36}$ ). The pulse-train excitation, with the duty cycle determined by the "dwell time" of the excitation beam on each bead, is: 1) inefficient due to over an order of magnitude reduction in time-averaged force exerted on each probe bead compared to continuous excitation ${ }^{36}$, and 2) does not readily support microrheological quantification of viscoelasticity ${ }^{37,38}$ due to the presence of higher harmonics in the comb-like excitation profile. Thus, a more efficient localized mechanical excitation scheme is needed in order to realize practical volumetric quantitative micromechanical imaging with PF-OCE. Inspired by the transformative impact of light-sheet microscopy for high-throughput bio-microscopy, we leverage photonic radiation pressure from a light-sheet, as opposed to a standard focused Gaussian beam, to achieve efficient parallel mechanical excitation. LS-pfOCE addresses the outstanding limitations of the original Gaussian-beam PF-OCE and dramatically improve the capability for quantitative volumetric time-lapse micromechanical imaging.

\section{Characterization and validation of the LS-pfOCE system}

The LS-pfOCE system adopts a pump-probe configuration. A weakly-focused light-sheet generated by a cylindrical lens is used for radiation-pressure excitation, instead of a Gaussian beam (Fig. 1a, see Supplementary Fig. 1 for a detailed system schematic). The light-sheet has a measured full-width half-maximum (FWHM) dimension of $80 \mu \mathrm{m}$ $\times 1.4 \mu \mathrm{m}$ at the focal plane (Fig. 1b) and a total beam power of $120 \mathrm{~mW}$ at the sample surface, providing a light-sheet radiation-pressure force with a peak of $\sim 3 \mathrm{pN}$ and a FWHM spatial extent of $80 \mu \mathrm{m}$ in the lateral (long-axis) and axial dimensions (Fig.1c, d). By harmonically modulating its power, the light-sheet PF beam can exert continuous sinusoidal radiation-pressure force on multiple probe beads located within the span of its long-axis in parallel, without the need for beam-scanning (Fig. 1a, inset). The radiation-pressure-induced bead oscillations are detected by a phasesensitive OCT, whose fast-axis scanning is parallel to and co-aligned with the long axis of the light-sheet, operating in a 2D BM-mode acquisition scheme at each slow-axis position (see Methods for specific acquisition parameters). Volumetric imaging was accomplished by translating the sample with a motorized actuator stage in a direction perpendicular to the long axis of the light-sheet (equivalent to the slow-axis scanning of the OCT system). Under this acquisition scheme, the light-sheet implementation provides a $7 \times$ improvement in the time-averaged radiationpressure force exerted on each probe bead over the equivalent Gaussian-beam implementation with the same PF beam power. The presented LS-pfOCE acquisition scheme supports quantitative 3D micromechanical imaging up to a maximum shear elastic modulus of 550-1200 Pa (with an experimental displacement sensitivity of 36-76 pm for OCT signal-to-noise ratio $\geq 28 \mathrm{~dB}$ ) over a volumetric field-of-view (FOV) of $80 \mu \mathrm{m} \times 350 \mu \mathrm{m} \times 80 \mu \mathrm{m}$ (fast-axis $\times$ slowaxis $\times$ depth), which typically contains several hundreds of embedded probe beads (the en-face FOV can be readily adjusted by extending the range of the slow-axis scan; see Methods and Discussion).

Light-sheet radiation-pressure-induced complex mechanical response of individual probe beads is measured via phase-sensitive OCT following compensation for the PT response of the medium. Compared to our previous work ${ }^{36}$, in order to improve the compatibility for live-cell imaging applications, LS-pfOCE implements a more robust PT response compensation approach that eliminates the need to incorporate exogenous PT reporters into the sample ${ }^{36}$. The micromechanical properties (i.e., the local complex shear modulus $G^{*}=G^{\prime}+i G^{\prime \prime}$ in the "microenvironment" of each bead) can be reconstructed from the measured bead-wise complex mechanical response and the experimentally measured light-sheet radiation-pressure force profiles (Fig. 1c, d; Supplementary Method 2 provides a detailed description the LS-pfOCE reconstruction procedure). Quantification of $G^{*}$ by LS-pfOCE is validated in homogeneous PAAm gels (see Methods for sample preparation) by comparison to parallel-plate shear rheometry (Fig. 1e). Under the premise that the mesh size of the PAAm polymer network is smaller than the size of LS-pfOCE probe beads (1.7- $\mu \mathrm{m}$ diameter), a micro-to-macroscale comparison can reasonably be made between the storage modulus, $G^{\prime}$, measured by LS-pfOCE and shear rheometry ${ }^{37}$. The measurement of $G^{\prime}$ by LS-pfOCE is in good agreement with that of shear rheometry at the same modulation frequency of $20 \mathrm{~Hz}$. However, LS-pfOCE measurement of the loss modulus, $G^{\prime \prime}$, differs from that of shear rheometry due to distinct viscous responses (e.g., viscous drag of fluid flow through the porous polymer network) at the micro- versus macroscale, which is in agreement with our prior PF-OCE work based on a Gaussian-beam excitation ${ }^{37,38}$. 


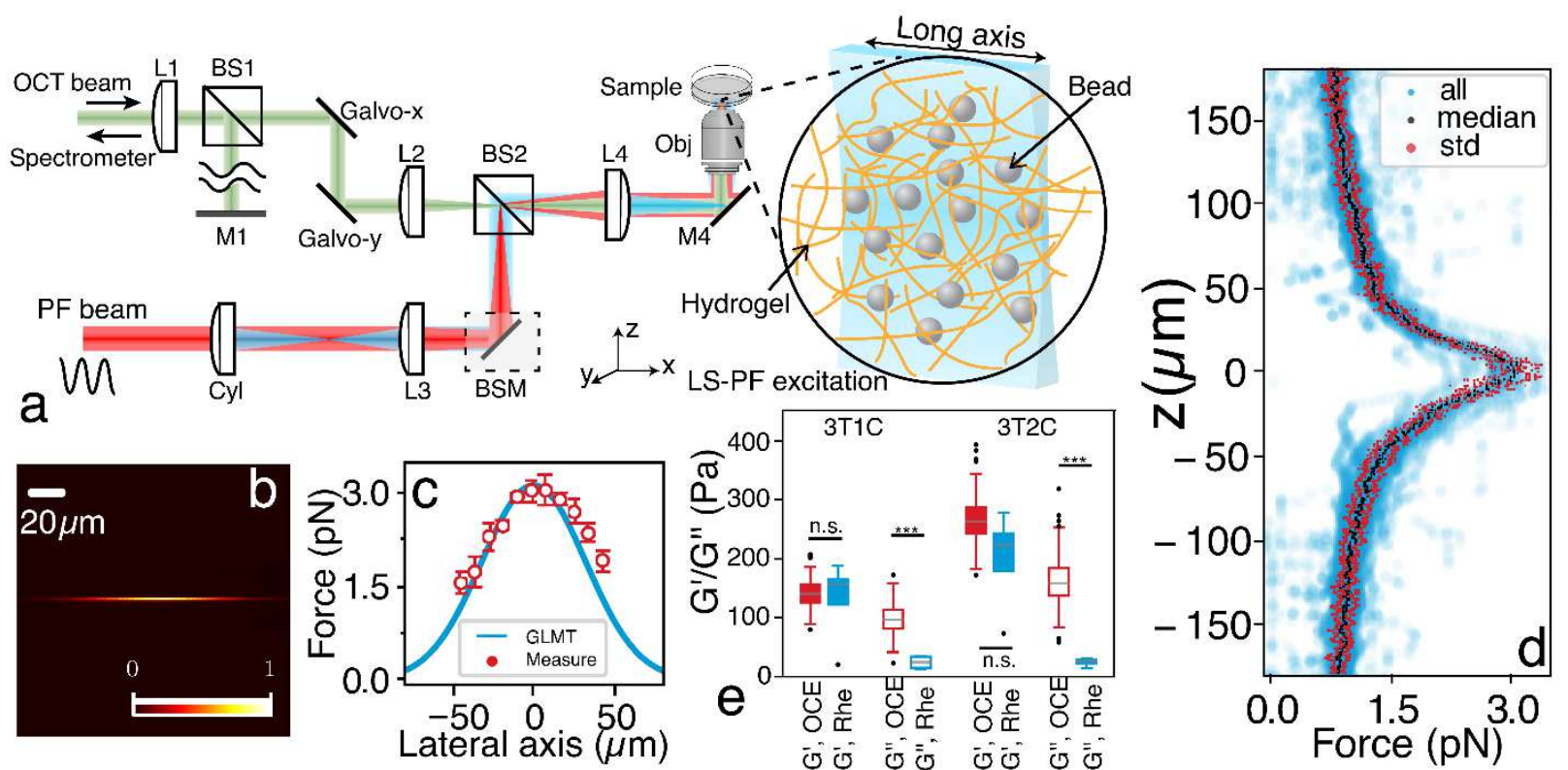

Fig. 1 LS-pfOCE system characterization and validation. a, Schematic of the LS-pfOCE system. Light-sheet radiation-pressure excitation is generated by a cylindrical lens and combined in free-space with the sample arm of a spectral-domain OCT system for interferometric detection of radiation-pressure-induced bead oscillations. See Methods for a full description of the LS-pfOCE system. L: lens, Cyl: cylindrical lens, BS: beam splitter, BSM: beamsteering module, M: mirror, Obj: microscope objective. b, Light-sheet intensity profile measured at the focal plane. c, Measured (red, mean \pm standard deviation from $N=48$ beads) and simulated (blue, Generalized Lorenz-Mie Theory (GLMT), see Supplementary Method 6) lateral light-sheet radiation-pressure profile at its focal plane on 1.9- $\mu \mathrm{m}$ melamine-resin beads. d, Measured axial light-sheet radiation-pressure profile at its lateral center on 1.9- $\mu \mathrm{m}$ melamine-resin beads, showing data (blue) and depth-dependent median (black) and median absolute difference (red) from $N=48$ beads. See Methods for light-sheet radiation-pressure force measurement procedure. e, Comparison of $G^{\prime}$ and $G^{\prime \prime}$ in PAAm gels measured by 3D LS-pfOCE (red) and bulk shear rheometry (blue) at $20 \mathrm{~Hz}$. $N=5$ samples for rheometry and $N=225$ and 263 beads for LS-pfOCE in 3T1C and 3T2C gels, respectively. Significant difference in group means is indicated by $* * * p$-value $<0.001$ and n.s. $p$-value $>0.05$.

\section{Imaging the micromechanical heterogeneity of fibrous ECM}

We demonstrate quantitative characterization of micromechanical heterogeneity in ECM constructs by performing LS-pfOCE in three microscopically heterogeneous fibrous collagen matrices with different microarchitectures (see Methods for sample preparation) and homogeneous PAAm hydrogel. LS-pfOCE is able to reveal the micromechanical heterogeneity of the fibrous collagen matrix compared to the homogeneous PAAm gel (Fig. 2a), where the distributions of both $G^{\prime}$ and $G^{\prime \prime}$ measured within a sample are broader (i.e., larger FWHM) for collagen than PAAm (Fig. 2b). LS-pfOCE measurements of $G^{\prime}$ (elasticity or "stiffness") and $R=G^{\prime \prime} / G^{\prime}$ (relative viscosity or "loss ratio") is also able to reflect the distinct microarchitectural characteristics of the three collagen matrices (Fig. 2c). The most microarchitecturally homogeneous collagen sample $\mathrm{C} 1$, with the thinnest collagen fibres, exhibits an overall lower stiffness and little variability in both $G^{\prime}$ and $R$. In contrast, the thicker fibrous samples $\mathrm{C} 2$ and $\mathrm{C} 3$ exhibit an overall higher stiffness with significant variability in both $G^{\prime}$ and $R$. Quantitatively, samples $\mathrm{C} 2$ and $\mathrm{C} 3$, with the more heterogeneous microarchitecture, have a broader distribution for both $G^{\prime}$ and $G^{\prime \prime}$ compared to that of sample $\mathrm{C} 1$ and the PAAm gels (Fig. 2d). 


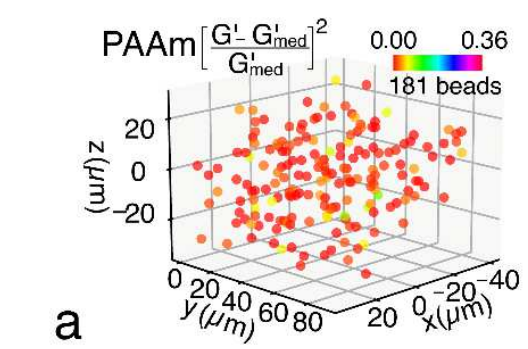

Confocal

\section{OCE-R}

C

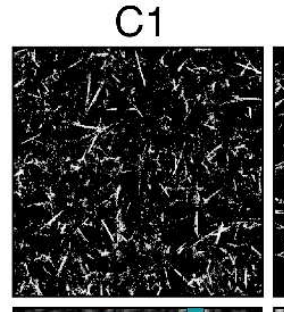

OCE-G'
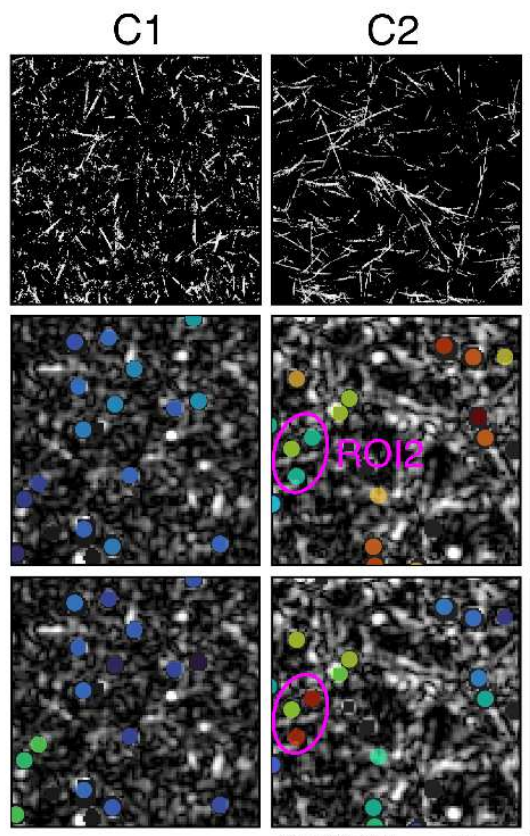

OCE colorbar \{

$\left\{\begin{array}{l}\mathrm{R} \\ 0.2 \text { (more solid-like) }\end{array}\right.$
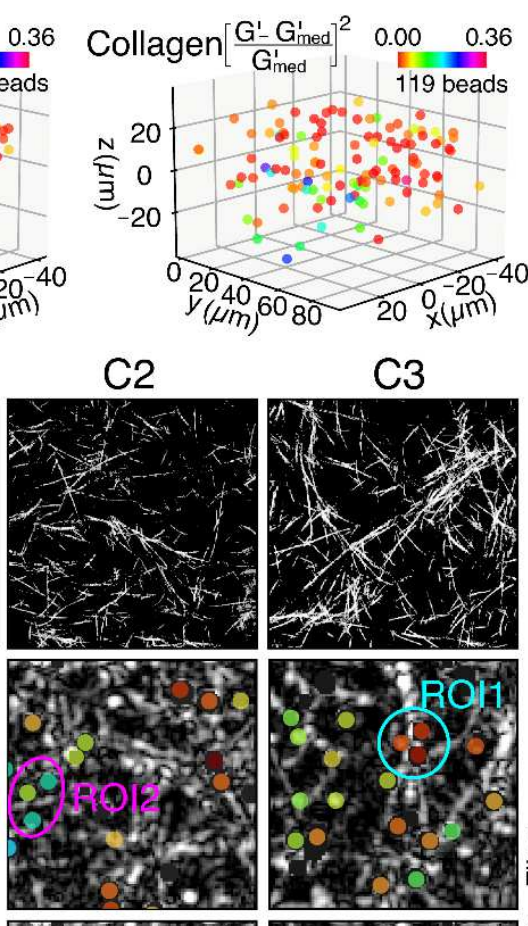

C3
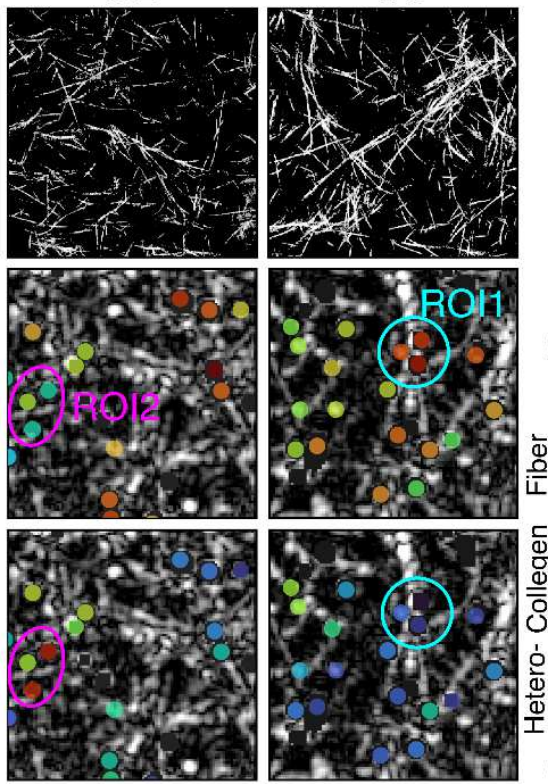

G'72.2(less stiff)

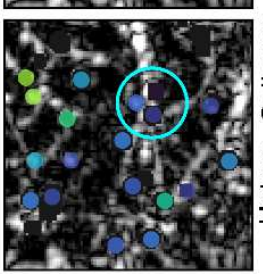

465.8 (more stiff)
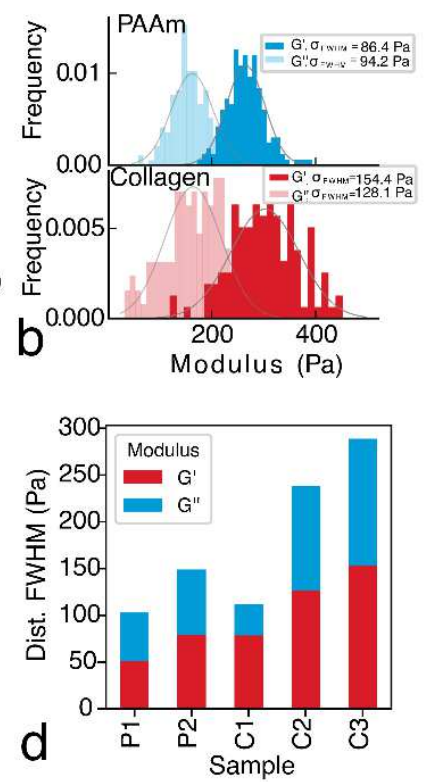

遂

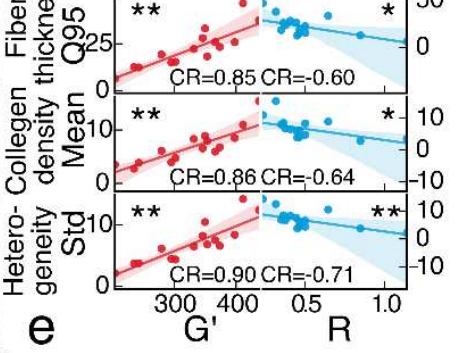

1.7(more fluid-like)

Fig. 2 Characterization of micromechanical heterogeneity in fibrous collagen matrices. a, Normalized relative variation in stiffness measured by 3D LS-pfOCE in a PAAm gel (3T2C, left) and collagen matrix (C3, right), where larger variation is observed in collagen. $N=181$ and 119 beads for PAAm and collagen, respectively. b, Histograms and statistical distributions of absolute (i.e., without normalization) $G^{\prime}$ and $G^{\prime \prime}$ measurements in a. $\sigma_{\text {FWHM }}$ indicates the FWHM of the distributions. c, En face confocal reflectance microscopy (top, FOV $106 \mu \mathrm{m} \times 106 \mu \mathrm{m}$ ) and OCT images overlaid with color-coded LS-pfOCE measurements of $G^{\prime}$ (middle) and $R$ (bottom) at the focal plane. ROI1 (cyan circles) indicates a stiffer and more solid-like microenvironment in the presence of thick collagen fibres. ROI2 indicates a more compliant and fluid-like microenvironment in the absence of any clear collagen fibres. All OCE images show FOV of $71 \mu \mathrm{m} \times 71 \mu \mathrm{m}$. d, FWHM of the distributions of LS-pfOCE $G^{\prime}$ (red) and $G^{\prime \prime}$ (blue) in PAAm gels and collagen matrices. $N=137,107,105,132$ and 100 beads for P1 (3T1C), P2 (3T2C), C1, C2, and C3, respectively. e, Correlation of LS-pfOCE $G^{\prime}$ (red) and $R$ (blue) to surrogate measures of collagen matrix microarchitectural characteristics. $N=15$ beads in sample C3. Q95, Mean, and Std indicate the 0.95 quantile, mean, and standard deviation of OCT scattering intensity within a 3- $\mu \mathrm{m}$ radial distance from the circumference of each bead, respectively (see Methods for further details). CR indicates the Spearman rank correlation coefficient; significance of the correlation is indicated by $* p$-value $<0.05$ and $* * p$-value $<0.01$. Solid lines and shaded regions represent linear fits and $95 \%$ confidence intervals, respectively.

The ability to microrheologically quantify viscoelasticity (i.e., both $G^{\prime}$ and $G^{\prime \prime}$ ) of LS-pfOCE also reveals an interesting observation: the variations in $G^{\prime}$ and $R$ tend to follow opposite trends in more fibrous $\mathrm{C} 2$ and $\mathrm{C} 3$ samples. For instance, region of interest (ROI) 1 (cyan circles in Fig. 2c), where the microenvironment is dominated by a junction of thick collagen fibres, exhibits higher stiffness (higher $G^{\prime}$ ) and a more "solid-like" behaviour (lower $R$ ). On the other hand, ROI2 (magenta circles in Fig. 2c), where the microenvironment is absent of any clearly resolvable 
collagen fibres, exhibits lower stiffness (lower $G$ ') and a more "fluid-like" behaviour (higher $R$ ). This observation may be supported by the biphasic description of a porous hydrogel, where the collagen fibres form the solid phase with an elastic response (which dominates in ROI1) and the rest of the material form the fluid phase with a viscous response (which dominates in ROI2). These results may also support the "network connectivity" interpretation of a biopolymer matrix $^{39-43}$ - ROI1 contains nodes with 3-6 connected collagen branches (thus, exhibiting higher node stiffness), whereas ROI2 contains nodes with $0-2$ connected branches at most (thus, exhibiting lower node stiffness). To corroborate these interpretations, LS-pfOCE measurement in sample C3 is correlated to three surrogate measures of the microarchitectural characteristics of the microenvironment of each bead (Fig. 2e): "Q95" for collagen fibre thickness, "Mean" for collagen density, and "Std" for matrix heterogeneity (see Methods for further details of this analysis). Both $G^{\prime}$ ' and $R$ show a strong (correlation coefficient $>0.85$ ) and statistically significant correlation to all three measures of the collagen microarchitectures. Overall, the results in Fig. 2 demonstrate the capability of LSpfOCE for quantitative micromechanical characterization of microscopically heterogeneous fibrous ECM constructs, which can enable correlative analysis between micromechanical and microarchitectural heterogeneities in the study of biopolymer mechanics.

\section{Imaging the cell-mediated ECM micromechanical remodelling}

We demonstrate potential mechanobiological applications of LS-pfOCE for live-cell imaging studies of cell-mediated ECM remodelling in 3D fibrin constructs seeded with NIH-3T3 fibroblasts (see Methods for sample preparation). LSpfOCE is able to quantitatively characterize, in $3 \mathrm{D}$, the spatial variations in the micromechanical properties of the fibrin ECM surrounding isolated cells (Fig. 3a, b). Higher $G^{\prime}$ can be observed closer to the cell body (especially extending from the tip of the cell) compared to regions further away, which corroborate previous results from OT$\mathrm{AMR}^{32,33}$. Quantitatively, both the mean $G^{\prime}$ value and the width of the distributions of $G^{\prime}$ and $G^{\prime \prime}$ measurements are larger in the cell-modified ECM (i.e., pericellular space) than the native (i.e., cell-free region) fibrin ECM (Fig. 3d). LS-pfOCE is also able to characterize changes in the pericellular micromechanical properties due to altered cellular activity. As an example, we investigated the consequences of reduced cellular contractility via treatment with Cytochalasin D (CytoD), an inhibitor of actin polymerization (Fig. 3c, measurement taken 2 hours after CytoD treatment, see Methods for the live-cell imaging protocol). Compared to the normal condition (Fig. 3b), the CytoDtreated sample exhibits strongly reduced stiffness in the pericellular space (especially at the cell tip) and the spatial distribution of $G^{\prime}$ appears relatively uniform (Fig. 3c). Significant differences are observed in both the group means and variances of $G^{\prime}$ and $G^{\prime \prime}$ measurements between the normal and CytoD conditions (Fig. 3e). Control experiments with pure DMSO (solvent for CytoD treatment) and measurements in blank fibrin constructs (not seeded with cells) are provided in Supplementary Data 1.

LS-pfOCE is also able to quantitatively characterize the variations in $G^{\prime}$ and $R$ as a function of distance to the cell body in 3D space (see Methods for details of this analysis). A decreasing trend in $G^{\prime}$ as a function of distance is apparent within the pericellular space (within $30 \mu \mathrm{m}$ of cell boundary) in both the normal and CytoD conditions (Fig. 3f, solid markers; and Supplementary Data 2). However, the stiffness gradient is significantly steeper in the normal condition (decay exponent of -0.19 for normal versus -0.08 for CytoD condition, see inset of Fig. $3 \mathrm{f}$ ), suggesting that the degree of cell-mediated pericellular stiffening is reduced (as expected) after the inhibition of actin polymerization. The weaker stiffness gradient that remains after CytoD treatment may be attributed to the accumulatively increased fibrous matrix density in the pericellular space (i.e., cell-mediated accumulation of fibrin during the 12-hr incubation period under normal conditions, prior to the treatment) ${ }^{33}$. Compared to $G^{\prime}$, the variation in $R$ as a function of distance to cell is less apparent and does not follow an obvious monotonic trend (Fig. 3f, open markers). However, a mild increasing trend can be observed within the pericellular space ( $\leq 30 \mu \mathrm{m}$ to cell), opposing the decreasing trend in $G^{\prime}$, in the normal condition.

The contributions of cell-force-mediated nonlinear ECM stiffening (i.e., strain- and/or stress-stiffening response of the fibrous ECM induced by cellular contractility) versus fibrous concentration-dependent ECM stiffening (i.e., accumulation of higher matrix density around cell) to the observed pericellular stiffness gradient have been speculated in a previous OT-AMR study, where similar results were observed after CytoD treatment ${ }^{33}$. Furthermore, our 3D LSpfOCE results also reveal a small increase in $G^{\prime}$ after the CytoD treatment in the intermediate ECM $(\geq 30 \mu \mathrm{m}$ away from the cell boundary in Fig. 3f, see Supplementary Data 3) - a small difference which could not be clearly discerned from previous bead-wise OT-AMR measurements ${ }^{33}$. We hypothesize that this mild stiffening in the intermediate ECM is related to the redistribution of the fibrin matrix - from where the matrix is concentrated by the contractile cell in the pericellular space ( $\leq 30 \mu \mathrm{m}$ to cell), back into the intermediate regions ( $\geq 30 \mu \mathrm{m}$ to cell) - following the inhibition of 
cellular contractility. Compared to the OT-AMR study ${ }^{33}$, LS-pfOCE facilitates a more complete, high-throughput volumetric characterization of cell-mediated spatial variations in the micromechanical properties of surrounding ECM by providing quantitative measurements of both elasticity and viscosity in 3D (as opposed to serially measuring a selected number of probe beads, typically within a 2D plane).

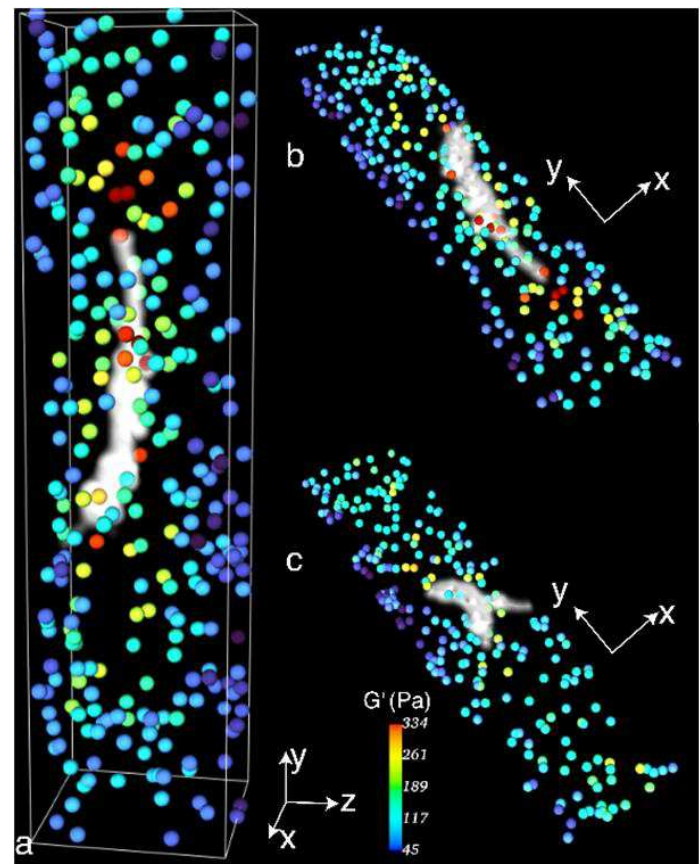

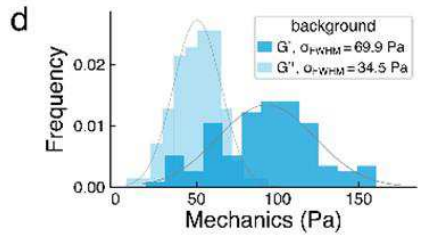
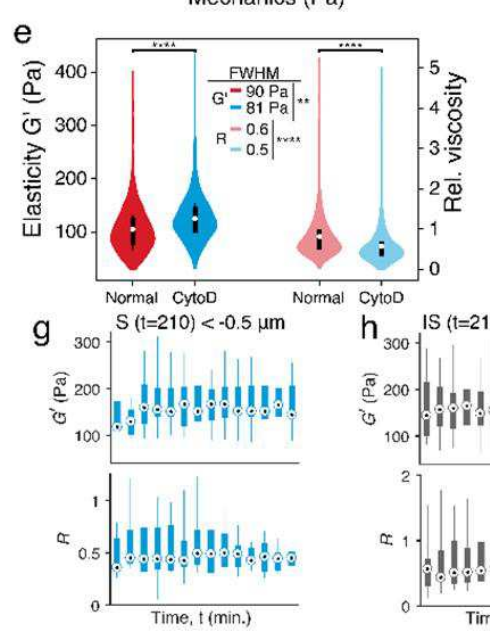

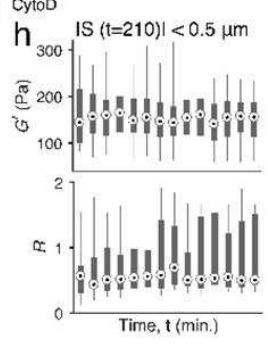

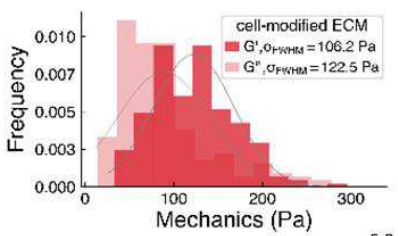

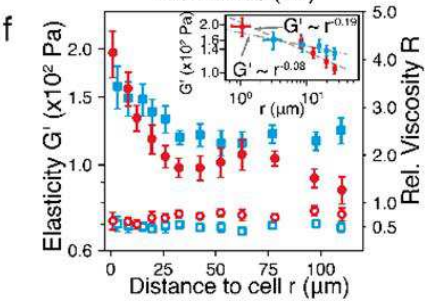

i $S(t=210)>0.5 \mu m$

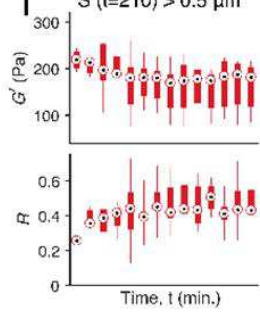

Fig. 3 Live-cell imaging of cell-mediated spatiotemporal variations in ECM micromechanical properties. a, 3D LS-pfOCE measurements of $G^{\prime}$ (color-coded beads) around a cell (white) and $z$-projection of 3D measurements under $\mathbf{b}$, normal condition and $\mathbf{c}, 2$ hours after treatment with CytoD (b and $\mathbf{c}$ show two different cells). Size of the beads is not to scale with the cell body. All images share the same colour scale. (See Supplementary Videos 1 and 2 for visualization of $\mathbf{b}$ and $\mathbf{c}$ from different viewing angles.) d, Histograms and distribution fits of 3D LS-pfOCE measurements in the pericellular (red, $N=205$ beads) and cell-free regions (blue, $N=97$ beads) from 1 cell. $\sigma_{\text {FWHM }}$ indicates the FWHM of the distributions. e, Violin plots of 3D LS-pfOCE measurements around cells under normal (red, $N=1824$ beads) and CytoD (blue, $N=1567$ beads) conditions from 8 cells. The FWHM of each distribution is stated. Significant difference in group means and variance is indicated by $* * p$-value $<0.01$ and $* * * * p$-value $<0.0001$. f, 3D LS-pfOCE measurements of $G^{\prime}$ (left) and $R$ (right) from the data in $\mathbf{e}$ as a function of distance to cell under normal (red) and CytoD (blue) conditions. Inset shows power law fits within $\leq 30 \mu \mathrm{m}$ to cell; the decay exponent has a $95 \%$ confidence interval of $-0.19 \pm 0.064$ (red) and $-0.08 \pm 0.024$ (blue). Each data point represents mean \pm standard deviation from $N \geq 20$ beads. $\mathbf{g}-\mathbf{i}$, Boxplots of $G^{\prime}$ (top) and $R$ (bottom) from 5 cells as a function of time after CytoD treatment in regions that experience high-negative (blue, $N=10$ beads), low (grey, $N=16$ beads), and high-positive (red, $N=8$ beads) matrix deformation $S$, respectively. Whiskers represent the full range of values.

LS-pfOCE also enables time-lapse monitoring of the spatiotemporal dynamics of micromechanical properties in live cellular systems. As an example, we investigated the temporal variations in the pericellular micromechanical properties (near the tip of the cell) as the CytoD treatment takes effect (see Methods for time-lapse imaging protocol). Time-lapse LS-pfOCE is able to simultaneously track the changes in both micromechanical properties and local matrix deformations (i.e., displacements of probe beads, see Methods for details of this calculation), which reveals statistically significant correlations between the former and the latter (Supplementary Data 4). This result is consistent with the hypothesis that the effect of CytoD treatment on pericellular micromechanical properties is governed by the spatiotemporal dynamics of the inhibition of actin polymerization, which in turn impacts cell-force-mediated matrix deformations.

Our time-lapse LS-pfOCE measurements also reveal an interesting behaviour that has never been reported by other techniques to our knowledge. While previous OT-AMR studies have only reported the softening of the ECM 
following contractility inhibition ${ }^{32,33}$, time-lapse LS-pfOCE shows that the changes in micromechanical properties and local matrix deformation occur in both positive and negative directions. Specifically, regions of the matrix that experience a negative deformation tend to exhibit an initially lower $G^{\prime}(\sim 100 \mathrm{~Pa}$, similar to the native fibrin in the cellfree regions in Fig. 3d) that increases over time (Fig. 3g). Conversely, regions of the matrix that experience a positive deformation tend to exhibit an initially higher $G^{\prime}(>200 \mathrm{~Pa}$, similar to the cell-modified pericellular fibrin in Fig. 3a, b) that decreases over time; this is also accompanied by an opposite trend in $R$ (Fig. 3i). The latter behaviour is consistent with previous reports ${ }^{32,33}$, and may be attributed to the "relaxation" of cell-force-mediated ECM stiffening governed by CytoD inhibition of actin polymerization ${ }^{44}$. Notably, both $G^{\prime}$ and $R$ stabilize after approximately $2 \mathrm{hr}$, consistent with the amount of time taken for the total cell force to approach zero following CytoD treatment in a previous traction force microscopy study ${ }^{44}$. The former behaviour, which has not been reported previously, may be attributed to the redistribution of fibrin network in the pericellular space (see discussion of Fig. 3f above) as well as the cell-mediated ECM stiffening by any residual actin filaments that have yet to be affected by CytoD (noting that attachment proteins on the cell surface to the ECM can remain even after the inhibition of actin polymerization).

Meanwhile, regions of the matrix that experience minimal deformation in either direction also tend to exhibit smaller changes in $G^{\prime}$ and an overall higher $R$ (Fig. 3h), which suggests that the microarchitecture of these regions may be dominated by large fluid-filled pores. Remarkably, all three types of dynamic responses stabilize to a similar level $\left(G^{\prime} \sim 160 \mathrm{~Pa}\right.$ and $R \sim 0.4$ ) over time after CytoD inhibition of cellular contractility, even though they initially exhibit distinct cell-mediated ECM micromechanical properties (Fig. 3g-i). Overall, the results in Fig. 3 demonstrate that the live-cell imaging capability of LS-pfOCE can provide access to previously unavailable measurements, which emphasizes its potential to support new discoveries in the field of mechanobiology.

\section{Discussion}

LS-pfOCE harnesses photonic radiation pressure from a light-sheet, and leverages label-free tomographic imaging via phase-sensitive OCT, to perform high-throughput quantitative 3D micromechanical imaging in biological hydrogels and live cellular systems. The unprecedented combination of light-sheet optical manipulation and state-of-the-art interferometric displacement measurements provides a foundation for future studies and development of photonic radiation pressure, including opening a new application area of light-sheet optics in elastography/microrheology. Compared to our previous work ${ }^{36-38}$, the dramatically improved imaging capabilities of LS-pfOCE rely on two key innovations - a parallel radiation-pressure force scheme that enables volumetric imaging with continuous harmonic excitation on each individual probe bead, and a more robust PT compensation approach that eliminates the need for exogenous PT reporters (see Methods). We applied LS-pfOCE to characterize the micromechanical heterogeneity in fibrous collagen matrices, and for the first time, characterized the 3D spatial variations and temporal dynamics of cellmediated ECM micromechanical properties via live-cell imaging of fibroblasts within 3D fibrin constructs. The 3D imaging of cell boundaries based on OCT speckle fluctuations ${ }^{6,44}$, as well as ECM deformations and micromechanical properties allows the measurements of pericellular mechanical properties to be interpreted within the context of the cell orientation (i.e., extension direction) and cell-induced ECM deformations.

Although the fast-axis scan range and depth coverage of the current LS-pfOCE system is limited by the lateral extent (long-axis width) and the NA (short-axis focusing) of the light-sheet, a larger volumetric FOV can be achieved by simply tuning the long-axis width of light-sheet PF beam. More sophisticated light-sheet generation optics can be introduced to generate laterally uniform light-sheet PF excitation with extended depth of focus, such as those previously developed for light-sheet fluorescence microscopy ${ }^{45-47}$. In addition, the volumetric throughput of LSpfOCE can be further improved by increasing the mechanical response amplitude-to-noise ratio ${ }^{37,38}$ (such as by increasing the power of the PF beam or reducing the OCT system noise), which would allow reliable measurements of bead mechanical response to be made with a lower number of temporal frames per slow-axis position ${ }^{37,38}$. One way to reduce noise in the current LS-pfOCE system is by replacing the motorized actuator with a high-fidelity stepper motor. Additional improvements may be realized by implementing common-path phase-sensitive detection ${ }^{48}$.

The acquisition scheme of LS-pfOCE can be flexibly tailored to best fit the experimental needs of specific mechanobiological applications - by adjusting the number of temporal (BM-mode) frames acquired at each slow-axis position. This navigates the trade-off between acquisition time and the maximum stiffness that can be reliably quantified (i.e., the displacement sensitivity supported by a given number of temporal frames ${ }^{37,38}$ ). Meanwhile, LSpfOCE may also be implemented with other high-speed OCT imaging approaches such as swept-source and parallel line-field OCT, in order to further improve its imaging $\operatorname{speed~}^{49}$. A faster option for OCT beam-scanning, such as a 
resonant scanner for multi-MHz-OCT ${ }^{50}$ or even a MEMS scanner ${ }^{49}$ (since LS-pfOCE does not require a large scanning angle to span the long axis of the light-sheet), that can support BM-mode frame rate in the $\mathrm{kHz}$ range will significantly improve the acquisition speed overall (see Supplementary Discussion 1 for further considerations related to high speed imaging).

LS-pfOCE has the potential to enable numerous novel research studies in the field of mechanobiology. Recent developments in traction force microscopy (TFM) have enabled the study of time-varying cell forces and cell-mediated ECM deformations in 3D, including both isolated ${ }^{8,44,51,52}$ and collective cellular behaviours (such as stromal-cellmediated dissemination of cancer cells from co-cultured tumour spheroids ${ }^{6,53,54}$ and epithelial-mesenchymal transitions in multicellular epithelial clusters ${ }^{55}$ ). LS-pfOCE has the potential to significantly elevate such studies by providing crucial (albeit currently missing) information on the 4D spatiotemporal dynamics of the ECM micromechanical properties (our correlative analysis of the changes in ECM viscoelasticity and matrix deformation in Fig. 3g-i and Supplementary Fig. 12 is an example of how new mechanobiological insights may be obtained with the combination of LS-pfOCE and TFM-based measurements). Furthermore, LS-pfOCE can be integrated into a multimodal microscopy platform that incorporates confocal or light-sheet fluorescence microscopy to enable simultaneous characterization of micromechanics and molecular signalling pathways.

Moreover, ongoing efforts in the field of biophysics are focused on the development of computational models to describe and unravel the complexities of biopolymer mechanics, including fibrous ECM constructs ${ }^{5,39-43}$. LS-pfOCE has the potential to help inform and validate the development of these computational models by providing experimental quantification of the micromechanical properties of biopolymer constructs (such as the fibrous collagen matrices in Fig. 2). To this end, future applications of LS-pfOCE may extend beyond the scope of linear viscoelasticity, to investigate poroelasticity and anisotropy (e.g., by rotating the sample to exert force from different directions) of fibrous biopolymer networks.

\section{Methods}

\section{LS-pfOCE system and beam alignment procedure}

The LS-pfOCE system consisted of a spectral-domain (SD)-OCT imaging system and a light-sheet for radiationpressure force excitation, which is combined into the OCT sample arm in a pump-probe configuration (see Supplementary Method 1 for detailed system schematic). The OCT system was sourced by a broadband superluminescent diode (Thorlabs, LS2000B) with a centre wavelength and a FWHM bandwidth of $1300 \mathrm{~nm}$ and 200 $\mathrm{nm}$, respectively. Spectral data was detected by a spectrometer (Wasatch Photonics, Cobra 1300) with a bandwidth of $245 \mathrm{~nm}$ and a 2048-pixel line-scan camera (Sensors Unlimited, GL2048). The sample arm utilized a double-pass illumination/collection configuration with an inverted 20x microscope objective (Olympus, LCPLN20XIR) with an NA of 0.45 . Telecentric beam-scanning was accomplished with a 2-axis galvanometer (Cambridge Technology, ProSeries $1,10 \mathrm{~mm}$ ) and a unit-magnification telescope, which imaged the galvanometer to the back focal plane of the objective. The transverse and axial resolutions (in air) of the OCT system were $2.3 \mu \mathrm{m}$ and $3.4 \mu \mathrm{m}$, respectively. The light-sheet pump beam was generated by a laser diode (Frankfurt Laser Company, FLU0786M250, HI780 fibre output) at a wavelength of $789 \mathrm{~nm}$ and a cylindrical lens (Thorlabs, ACY254-100-B). The light-sheet was injected into the telescope of the OCT system via a longpass dichroic mirror (Thorlabs, DMLP1180R) and shared the same objective. The long axis of the light-sheet was collimated with a FWHM beam width of $80 \mu \mathrm{m}$ while the short axis was focused to a FWHM beam width of $1.4 \mu \mathrm{m}$. The power of the light-sheet pump beam was harmonically modulated with a peak power of $120 \mathrm{~mW}$ measured directly after the objective. 3D imaging was accomplished via galvanometer scanning of the OCT beam along the fast axis and a motorized actuator (Thorlabs, ZST225B motor, KST101 controller, and $\mathrm{KCH} 601$ controller hub/power supply) stepping the sample along the slow axis, while the light-sheet pump beam remained stationary.

A beam steering module ensured co-alignment between the pump and probe beams such that 1) the optical axes of both beams were parallel and overlapped, 2) the focal planes of both beams were $\leq 10 \mu \mathrm{m}$ from each other inside the sample, and 3) the long axis of the light-sheet was parallel to the fast galvanometer scanning axis of the OCT system. The beam alignment was visualized in real-time by placing a CCD camera (Thorlabs, DCC1545M) above the objective to image the light-sheet and OCT beam spots at different axial position relative to the focal plane. After the real-time alignment, the light-sheet radiation-pressure force was measured as a final LS-pfOCE system performance check prior to each experiment. 


\section{LS-pfOCE radiation-pressure force measurement}

The measurement of radiation-pressure force from the light-sheet followed a previously described OCT-based depthresolved force measurement method ${ }^{56}$, with modifications to extend the previous $1 \mathrm{D}$ measurement to $2 \mathrm{D}$ in order to characterize both the axial and lateral (along light-sheet long axis) force profiles (Fig. 1b, d). A 10\% w/w solution of glycerol and water (refractive index 1.3469, close to those of hydrogels, see Table 1 in Ref. ${ }^{56}$ for other relevant physical properties) was used as the viscous fluid medium for the force measurements. The $2 \mathrm{D}$ measurement was accomplished via a BM-mode acquisition scheme (instead of M-mode in Ref. ${ }^{56}$ ), with the fast-axis scanning parallel to the long axis of the light-sheet. The 'M-mode-equivalent' space-time axial bead trajectory images were obtained across the BM-mode frames (i.e., temporal dimension) at different lateral segments along the fast axis (i.e., long axis of light-sheet). The depth-resolved axial radiation-pressure force profile at each lateral segment was reconstructed by tracking the instantaneous axial velocity and acceleration of each bead from the space-time axial bead trajectory image, then, solving the $1 \mathrm{D}$ equation of motion along the axial direction using known fluid viscosity and other physical properties, as previously described ${ }^{56}$. This procedure is described in full in Supplementary Method 4. The final output is a $2 \mathrm{D}$ light-sheet radiation-presser force profile $F_{\text {rad }}(x, z)$.

Another modification from the previously described method was the implementation of a rapid automated algorithm based on Radon transform to extract depth-resolved bead axial velocity and acceleration from the spacetime bead trajectory images. The automated algorithm significantly reduced the time and labour that would otherwise be required to perform the coarse manual bead trajectory tracking over the entire 2D BM-mode datasets (200-400 beads total). This was essential to streamline the routine system alignment and force measurement procedure prior to each experiment. The full description of the Radon-transform-based automated force measurement algorithm is provided in Supplementary Method 5.

\section{LS-pfOCE data acquisition procedure}

Volumetric imaging with LS-pfOCE adopted a 3D BM-mode acquisition scheme ${ }^{36}$, with the modification that slowaxis scanning was accomplished by translating the sample with a motorized stage (instead of galvanometer scanning as typically done for 3D-OCT data acquisition). For all LS-pfOCE results presented here, a BM-mode frame rate of $425 \mathrm{~Hz}$ was implemented with a radiation-pressure force modulation frequency of $20 \mathrm{~Hz}$, which ensured sufficient temporal sampling per modulation cycle to reliably measure the phase shift w.r.t. to the drive waveform of the bead response. The fast-axis scan range was kept at $90 \mu \mathrm{m}$ to match the width (long axis) of the light-sheet. A total of 6400 BM-mode frames were acquired at each slow-axis position, supporting a theoretical (SNR-limited shot-noise) ${ }^{36,57}$ and experimental displacement sensitivity of $27 \mathrm{pm}$ and $76 \mathrm{pm}$ at an OCT SNR of $28 \mathrm{~dB}$, respectively. The lateral pixel size was $0.75 \mu \mathrm{m} \times 0.75 \mu \mathrm{m}$, which ensured that each probe bead (diameter of $1.7 \mu \mathrm{m}$ for polystyrene and $1.9 \mu \mathrm{m}$ for melamine-resin) were sampled at multiple lateral pixels. These acquisition parameters result in an acquisition time of 15 seconds per slow-axis position, which would total to 117 minutes for a slow-axis scan range of $350 \mu \mathrm{m}$ implemented in Fig. 3a-c. However, due to the delayed stabilization after stepping of our current motorized actuator (Thorlabs, ZST225B), we implemented a wait time of 6 seconds before initiating the acquisition at each slow-axis position. This significant wait time would not be necessary with a high-fidelity stepper motor. All instrument control and synchronization were accomplished with a custom LabVIEW (2014 64-bit version) acquisition software.

\section{LS-pfOCE reconstruction procedure}

The reconstruction of micromechanical properties of the medium in the vicinity of each probe bead from the raw 3D BM-mode LS-pfOCE data followed the previously describe quantitative procedure ${ }^{37}$, with modifications to implement the processing routine at multiple spatial voxels in the volumetric datasets (as opposed to 1D M-mode datasets in Ref. ${ }^{4}$ ) and accommodate for the excitation by the light-sheet (instead of Gaussian) pump beam. The full description of the LS-pfOCE reconstruction procedure is provided in Supplementary Method 2 together with a flowchart that outlines the full procedure (Supplementary Fig. 2). Briefly, the LS-pfOCE reconstruction procedure can be divided into the following 6 steps.

1) OCT image reconstruction, which implemented Fourier-domain OCT reconstruction and computational image formation procedure ${ }^{44}$ for defocus correction along the fast axis to obtain the complex 3D BM-mode OCT image, $\tilde{S}(x, y, z, t)$. 
2) Phase-sensitive OCE reconstruction, which implemented the procedure in Ref. ${ }^{37}$ to extract the optical path length (OPL) response from the phase of $\tilde{S}(x, y, z, t)$, after phase registration. The OPL response as a function of time at each spatial voxel is described as a complex phasor with amplitude $A(x, y, z)$ and phase shift $\varphi(x, y, z)$ w.r.t. to the drive waveform (for brevity, subsequent mention of "phase shift" shall refer to the phase shift w.r.t. to the drive waveform).

3) Image segmentation, which assigned the OPL response at each spatial voxel to either the PT response or the total response data region based on OCT image magnitude thresholds (values provided in Supplementary Method 2) and data exclusion criteria (indicated on Supplementary Fig. 2$)^{37}$.

4) Photothermal (PT) response reconstruction, which reconstructed the $2 \mathrm{D}$ PT response amplitude $A_{\mathrm{PT}}(x, z)$ and phase shift $\varphi_{\mathrm{PT}}(x, z)$ from the OPL response at the spatial voxels assigned to the PT response data region in step 3 .

5) Isolation of bead mechanical response, which subtracted the PT response reconstructed in step 4 from the OPL response at the spatial voxels assigned to the total response data region to isolate the mechanical response with amplitude $A_{\text {mech }}(x, y, z)$ and phase shift $\varphi_{\text {mech }}(x, y, z)$ at each spatial voxel. Then, the individual spatial voxels were clustered into their respective beads to compute the median mechanical response of each bead $A_{\text {mech }}\left(\mathbf{r}_{\mathrm{c}}\right)$ and $\varphi_{\text {mech }}\left(\mathbf{r}_{\mathrm{c}}\right)$, where $\mathbf{r}_{\mathrm{c}}=\left(x_{\mathrm{c}}, y_{\mathrm{c}}, z_{\mathrm{c}}\right)$ denotes the spatial coordinates of the bead centroid.

6) Complex shear modulus reconstruction, which solved the $1 \mathrm{D}$ axial equation of motion of a sphere forced into an oscillatory motion, described by an amplitude $A_{\text {mech }}\left(\mathbf{r}_{\mathrm{c}}\right)$ and phase shift $\varphi_{\text {mech }}\left(\mathbf{r}_{\mathrm{c}}\right)$, in a viscoelastic medium by an external harmonically-varying force with amplitude $F_{\text {rad }}\left(\mathbf{r}_{c}\right)$, extracted from the measured force profile $F_{\text {rad }}(x, z)$. The complex shear modulus of the medium in the vicinity of each bead $G^{*}\left(\mathbf{r}_{\mathrm{c}}\right)$ is given by:

$$
\tilde{G}_{\text {eff }}\left(\mathbf{r}_{\mathrm{c}}\right)=\frac{F_{\text {rad }}\left(\mathbf{r}_{\mathrm{c}}\right)+m \omega^{2}\left[A_{\text {mech }}\left(\mathbf{r}_{\mathrm{c}}\right) \exp \left(i \varphi_{\text {mech }}\left(\mathbf{r}_{\mathrm{c}}\right)\right)\right]}{6 \pi a\left[A_{\text {mech }}\left(\mathbf{r}_{\mathrm{c}}\right) \exp \left(i \varphi_{\text {mech }}\left(\mathbf{r}_{\mathrm{c}}\right)\right)\right]},
$$

where $\omega, m$, and $a$ denote the angular modulation frequency, mass, and radius of the oscillating sphere, respectively. The parameter $\tilde{G}_{\text {eff }}\left(\mathbf{r}_{\mathrm{c}}\right)$ is a function of $G^{*}\left(\mathbf{r}_{\mathrm{c}}\right)$ and is given by Oestreicher's model of an oscillating sphere in a viscoelastic medium, described by a complex shear wave number $k^{*}$, according to:

$$
\tilde{G}_{\text {eff }}\left(\mathbf{r}_{\mathrm{c}}\right)=G^{*}\left(\mathbf{r}_{\mathrm{c}}\right)\left[1-i k^{*}\left(\mathbf{r}_{\mathrm{c}}\right) a-\frac{1}{9} k^{* 2}\left(\mathbf{r}_{\mathrm{c}}\right) a^{2}\right]
$$

which can be inverted to obtain $G^{*}\left(\mathbf{r}_{\mathrm{c}}\right)$.

All data processing was implemented in MATLAB 2017a.

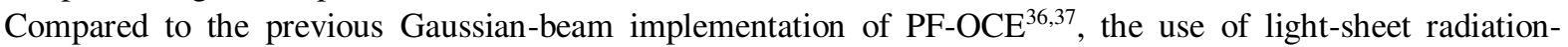
pressure force excitation has the largest impact on steps 4 and 6 listed above. This is due to the non-uniform lateral intensity profile of the light-sheet along its long axis (Fig. 1c, d), which causes both the radiation-pressure force and the PT response to vary not only as a function of depth (axial) but also across the fast axis (lateral along the long axis of light-sheet). Supplementary Method 4 provides a full description of the LS-pfOCE force reconstruction procedure. For the PT response, another important distinction implemented here compared to previously ${ }^{36,37}$ was that no exogenous PT reporters were added to the sample. This was done to improve the biocompatibility of LS-pfOCE for mechanobiological applications with live-cell imaging studies. The LS-pfOCE experiments presented here relied entirely on the intrinsic OCT scattering signal of the medium and the cumulative PT response measured at the coverslip (see Supplementary Fig. 3 the sample configuration) to reconstruct the full 2D profiles of the PT response amplitude and phase shift (Supplementary Fig. 4). Supplementary Method 3 provides a full description of the PT response 
calibration procedure for PAAm gels (Figs. 1e and 2a, b, d), collagen matrices (Fig. 2), and live-cell imaging in cellseeded fibrin constructs (Fig. 3).

\section{Sample preparation}

All samples were prepared in \#1.5 glass-bottomed petri dishes (MatTek, P35G-1.5-10-C), where the OCT beam interrogated the sample through the glass bottom (Supplementary Fig. 3). For the cell-free samples (i.e., PAAm gels and collagen matrices), a coverslip (Fisherbrand, 12540A) was placed on top of the sample well to provide measurements of the cumulative PT responses ${ }^{36,37}$. For live-cell imaging, the sample petri dish was placed in a stagetop incubated bio-chamber (Okolab, UNO-PLUS) for environmental control.

PAAm gels (Figs. 1e and 2a, b, d) were prepared as previously described ${ }^{37}$. Briefly, acrylamide monomer (BioRad, 40\% Acrylamide Solution, 1610140), bis-acrylamide crosslinker (Bio-Rad, 2\% Bis Solution, 1610142), and deionized water were mixed at appropriate concentrations (see Table 1 in Ref. ${ }^{37}$ for the compositions of 3T1C and 3T2C gels). An aqueous suspension of 1.7- $\mu \mathrm{m}$ diameter polystyrene beads (Spherotech, PP-15-10) was added to the mixture at a concentration of $30 \mu \mathrm{L} / \mathrm{mL}$ to achieve a mean particle separation of $12 \mu \mathrm{m}$. The solution was mixed and desiccated for $15 \mathrm{~min}$. PAAm polymerization was activated with $10 \%$ ammonium persulfate (Bio-Rad, APS, 1610700) as the redox initiator and tetramethylethylenediamine (Bio-Rad, TEMED, 1610800) as the catalyst. 10\% APS and TEMED were added at concentrations of $10 \mu \mathrm{L} / \mathrm{mL}$ and $1 \mu \mathrm{L} / \mathrm{mL}$, respectively. Hydrogels were allowed to polymerize for $60 \mathrm{~min}$ prior to LS-pfOCE measurements.

Collagen matrices (Fig. 2) were prepared with rat tail type I collagen (Corning, 354236) at a final collagen concentration of $2.0 \mathrm{mg} / \mathrm{mL}$. An aqueous suspension of $1.9-\mu \mathrm{m}$ diameter carboxyl-functionalized melamine-resin beads (microParticles GmbH, MF-COOH-S1000) was added to the mixture at a concentration of $19 \mu \mathrm{L} / \mathrm{mL}$ to achieve a mean particle separation of $14 \mu \mathrm{m}$. Melamine-resin beads were used here instead of polystyrene beads because they generated larger radiation-pressure force magnitude due the higher refractive index. Carboxyl functionalization ensured that the probe beads adhered to the collagen matrix rather than freely floating inside the pores. Three polymerization protocols were implemented to form collagen matrices with different fibre architecture. The $\mathrm{C} 1$ sample was polymerized at $37^{\circ} \mathrm{C}$ for $45 \mathrm{~min}$ to form a relatively uniform distribution of fine collagen fibre network. The $\mathrm{C} 2$ sample was polymerized at $22^{\circ} \mathrm{C}$ (room temperature) for $45 \mathrm{~min}$ to promote formation of thicker collagen fibres with more heterogeneous distribution. The $\mathrm{C} 3$ sample was polymerized at $4{ }^{\circ} \mathrm{C}$ for $15 \mathrm{~min}, 20^{\circ} \mathrm{C}$ for $15 \mathrm{~min}$, then $37^{\circ} \mathrm{C}$ for $15 \mathrm{~min}$ to form the most heterogeneous fibre architecture with the thickest collagen fibres ${ }^{6}$.

Cell-seeded fibrin constructs consisted of NIH-3T3 fibroblasts in 3D fibrin hydrogels. The cells were maintained in tissue culture flasks with media consisting of Dulbecco's Modified Eagle Medium (DMEM, Life Technologies, 11965092) supplemented with $10 \%$ fetal bovine serum (FBS, Life Technologies, 16170086) and 1\% penicillinstreptomycin (PS, Life Technologies, 15140122). Fibrin hydrogels were prepared with bovine fibrinogen (SigmaAldrich, F8630) solution in DMEM without serum and antibiotics, at a final concentration of $2.5 \mathrm{mg} / \mathrm{mL}$. NIH-3T3 cells and 1.9- $\mu \mathrm{m}$ diameter carboxyl-functionalized melamine-resin beads (microParticles GmbH, MF-COOH-S1000) were added to the fibrinogen solution to achieve a final cell density of $7 \times 10^{4}$ cells $/ \mathrm{mL}$ and an average bead spacing of $20 \mu \mathrm{m}$, respectively. Lower bead density was utilized here compared to in the collagen matrices to keep the volume fraction of exogenous beads below $1 \times 10^{-4}$ for live-cell imaging. The mixture was deposited into the sample well of a glass-bottomed petri dish, where polymerization into fibrin was initiated by $2 \mathrm{U}$ of bovine thrombin (Sigma-Aldrich, T4648) pre-aliquoted into the well. The cell-seeded constructs were incubated at room temperature for 5 min followed by $37{ }^{\circ} \mathrm{C}$ for $25 \mathrm{~min}$, then, rehydrated with DMEM containing $10 \%$ FBS and $1 \%$ PS. The LS-pfOCE measurements were performed after $12 \mathrm{hr}$ of incubation at $37^{\circ} \mathrm{C}$.

\section{Live-cell imaging protocols}

The cell-seeded fibrin constructs were incubated for $12 \mathrm{hr}$ prior to the LS-pfOCE measurements to allow for the cells to naturally spread. In the post-CytoD treatment condition, $50 \mu \mathrm{L}$ of a $100-\mu \mathrm{M}$ Cytochalasin D (Sigma-Aldrich, C2618) dissolved in DMSO was administered to the cell-seeded 3D fibrin construct and incubated at $37^{\circ} \mathrm{C}$ for another $2 \mathrm{hr}$. Immediately before LS-pfOCE measurements, the sample petri dish was placed inside the stage-mounted incubated bio-chamber. For 3D LS-pfOCE measurements (Fig. 3a-f), a cell with an elongated morphology and roughly oriented along the slow-axis scanning direction was selected for imaging. Guided by a real-time display of the en face OCT image, the cell was roughly positioned at the centre of the $80 \mu \mathrm{m} \times 350 \mu \mathrm{m}$ (fast axis $\times$ slow axis) transverse FOV by 
translating the sample stage. This FOV allowed the spatial variation in the pericellular space to be captured around the cell, with an extended distance on either side of the cell tips (i.e., major elongated axis of the cell).

For dynamic monitoring of the response to CytoD treatment (Fig. 3g-i), time-lapsed LS-pfOCE was performed 15 min after adding $20 \mu \mathrm{L}$ of the $100-\mu \mathrm{M}$ CytoD to the dish. An elongated cell was likewise located, but instead of positioning the cell at the centre of the FOV, a tip of the cell was positioned adjacent to an $80 \mu \mathrm{m} \times 26 \mu \mathrm{m}$ (fast axis $x$ slow axis) transverse FOV along the slow axis. This FOV allowed the spatiotemporal variations in the pericellular space adjacent to the cell tip to be captured every $15 \mathrm{~min}$ while the CytoD treatment was taking effect. Two control experiments were performed. For the blank control with CytoD (Supplementary Fig. 9a), 3D fibrin constructs were prepared as described above but without seeding the cells. First, a 3D LS-pfOCE dataset was acquired, then, the CytoD treatment was added (50 $\mu \mathrm{L}$ of a $100-\mu \mathrm{M}$ CytoD in DMSO solution) before another 3D LS-pfOCE dataset was acquired at approximately the same location. For the cell control with DMSO (Supplementary Fig. 9b), a 3D LSpfOCE dataset was acquired around a cell as described above. Then, $50 \mu \mathrm{L}$ of pure DMSO (without CytoD) was added and the sample was left in the bio-chamber for $2 \mathrm{hr}$ before another 3D LS-pfOCE dataset was acquired around the same cell.

\section{Parallel-plate shear rheometry of polyacrylamide gels}

Bulk complex shear modulus of each PAAm gel in Fig. 1e was measured with a parallel-plate shear rheometer (TA Instruments, DHR-3) using a 20-mm diameter plate, as described in Ref. ${ }^{37}$. Polymerization was achieved directly on the rheometer plate by pipetting $200 \mu \mathrm{L}$ of the activated polymer solution (i.e., after adding $10 \%$ APS and TEMED) onto the bottom plate. The gap was set $500 \mu \mathrm{m}$ and the excess polymer solution was carefully removed. The sample was sealed on the side with mineral oil to prevent evaporation. Polymerization was monitored in a time-sweep oscillatory test at oscillation frequency of $1 \mathrm{rad} / \mathrm{s}$ and shear strain of $0.5 \%$. All samples were left to polymerize for 60 minutes, during which the stabilization of shear moduli was confirmed. Then, a frequency-sweep oscillatory test was performed at the oscillation frequency ranging from $1-50 \mathrm{~Hz}$ and applied torque of $10 \mu \mathrm{N} \cdot \mathrm{m}$. A total of 5 measurements were made for each PAAm concentration.

\section{Confocal reflectance microscopy of collagen matrices}

The confocal reflectance images in Fig. 2c were obtained with a Zeiss LSM 710 laser-scanning confocal microscope, operating in the reflection mode at $488 \mathrm{~nm}$ with C-Apochromat 40x/1.2 water-immersion objective. The images were taken at a transverse FOV of $106 \mu \mathrm{m} \times 106 \mu \mathrm{m}$ with transverse resolution of $250 \mathrm{~nm}$. Each image was averaged over 16 en face planes. The collagen fibrous structures were then segmented by thresholding at 80 percentile of pixel values in each image. To further enhance the collagen contrast, the processed images were gamma-corrected with a coefficient of 0.4. All shown confocal images share the same grey colour scale. Image data was processed in Python v3.7.7.

\section{Analysis of micromechanical heterogeneity in collagen matrices}

The micromechanical heterogeneity measured in sample C3 by LS-pfOCE was correlated to the microstructural architecture of the collagen matrix in Fig. 2e. Three OCT image metrics were computed within a local volume encompassing a $3-\mu \mathrm{m}$ radius around each bead to serve as the surrogate measures of the microstructural characteristics of the bead microenvironment. First, the probe beads were removed from the 3D OCT image via a magnitude-based segmentation and dilation of the binary mask. Then, spatial voxels that were located $\leq 3 \mu \mathrm{m}$ distance away from the nearest circumference of each bead were collected. Three statistical metrics were computed from the collection of spatial voxels around each bead:

1) 0.95 quantile of OCT intensity ("Q95” in Fig. 2e), 2) provided a surrogate measure for collagen fibre thickness since thicker fibre generates higher OCT scattering intensity,

2) mean OCT intensity ("Mean" in Fig. 2e) provided a surrogate measure for local collagen density since the presence of more collagen fibres generated an overall higher OCT scattering intensity,

3) standard deviation of OCT intensity ("Std" in Fig. 2e) provided a surrogate measure for collagen matrix heterogeneity (e.g., a porous microenvironment next to a thick fibre would produce a large Std of the OCT intensity).

Spearman rank correlation coefficients and $p$-values between the bead-wise $G^{\prime}$ and $R$ measured by LS-pfOCE and the three local image metrics are indicated on Fig. 2e. (Although linear fit lines were plotted to guide the visualization of 
the trends, the reported correlation results represent the Spearman rank correlation and not the linear regression.) All analysis was implemented in MATLAB 2017a.

\section{Analysis of cell-mediated spatiotemporal variations in ECM micromechanical properties}

For the analysis of spatial variations in the micromechanical properties in the pericellular space (Fig. 3f), the distance $r$ from each probe bead to the cell body was defined as the length of the shortest line connecting the centroid of the bead to a point on the surface of the cell. The cell body (i.e., white cell structure in Fig. 3a-c) was segmented from the 3D BM-mode image with a previously described method based on temporal speckle contrast to distinguish the dynamic cellular structures from the static fibrin background ${ }^{37}$. (Here, standard deviation of OCT magnitude image was computed across temporal BM-mode frames instead of the 'burst' of 8 OCT volumes in Ref. ${ }^{37}$.) To calculate $G^{\prime}$ and $R$ as a function of $r$, the beads were grouped based on the distance $r$, with each group spanning $3 \mu \mathrm{m}$. The error bars were calculated as $95 \%$ confidence interval for the mean value in each distance group with Student's $t$-distribution. The curve fit in the inset of Fig. $3 \mathrm{f}$ was conducted by least square polynomial fit over the domain of $r \leq 30 \mu \mathrm{m}$. This portion of the analysis was implemented in Python v3.7.7.

For time-lapse monitoring immediately after adding the CytoD treatment, the cumulative bead displacement $S$ reported on Fig. $3 \mathrm{~g}-\mathrm{i}$ was computed from the spatial coordinates of the bead centroid $\mathbf{r}_{\mathrm{c}}=\left(x_{\mathrm{c}}, y_{\mathrm{c}}, z_{\mathrm{c}}\right)$ at each time point, after correcting for the bulk sample shift. The $x$ component of the cumulative displacement of the $m^{\text {th }}$ bead at the $n^{\text {th }}$ time point was computed from:

$$
\Delta x_{\mathrm{c}}\left(t_{n} ; m\right)=\sum_{i=2}^{n}\left[\left[x_{\mathrm{c}}\left(t_{i} ; m\right)-x_{\mathrm{c}}\left(t_{i-1} ; m\right)\right]-\frac{1}{N} \sum_{j}^{N}\left[x_{\mathrm{c}}\left(t_{i} ; j\right)-x_{\mathrm{c}}\left(t_{i-1} ; j\right)\right]\right]
$$

where $N$ denotes the total number of beads in the field-of-view. The inner summation term represents the bulk shift, estimated as the mean displacement of all beads between each adjacent time points. The $y$ and $z$ components of the cumulative bead displacement were computed in the same manner. The signed cumulative displacement of the $m^{\text {th }}$ bead at the $n^{\text {th }}$ time point was computed from the displacement vector $\Delta \mathbf{r}_{\mathrm{c}}=\left(\Delta x_{\mathrm{c}}, \Delta y_{\mathrm{c}}, \Delta z_{\mathrm{c}}\right)$ by:

$$
S\left(t_{n} ; m\right)=\operatorname{sgn}\left[\Delta_{\text {major }}\left(t_{n} ; m\right)\right]\left\|\Delta \mathbf{r}_{\mathrm{c}}\left(t_{n} ; m\right)\right\|,
$$

where $\operatorname{sgn}\left[\Delta_{\text {major }}\right]$ denotes the sign of the major $\Delta \mathbf{r}_{\mathrm{c}}$ component (i.e., $\Delta x_{\mathrm{c}}, \Delta y_{\mathrm{c}}$ or $\Delta z_{\mathrm{c}}$ ), defined as the largest component of the directional displacement vector, $\left(\left|\Delta x_{\mathrm{c}}\right|,\left|\Delta y_{\mathrm{c}}\right|,\left|\Delta z_{\mathrm{c}}\right|\right) /\left\|\Delta \mathbf{r}_{\mathrm{c}}\right\|$, averaged across all beads and time points. The beads were divided into three groups based on their $S$ values at the last time point to visualize the temporal variations of $G^{\prime}$ and $R$ in Fig. $3 \mathrm{~g}-\mathrm{i}$. This portion of the analysis was implemented in MATLAB 2017a.

\section{Statistical analysis}

For PAAm gels, Welch's $t$-test was implemented to test the difference between group means of LS-pfOCE measurements and shear rheometry measurements in both 3T1C and 3T2C PAAm gels in Fig. 1e. For collagen matrices, two-tail Spearman rank correlation was computed to test the correlation between LS-pfOCE measurements and local OCT image metrics in sample C3. The correlation was considered significant at $95 \%$ confidence level; the $p$-values are reported on Fig. 2e. For 3D live-cell imaging study, Welch's $t$-test for equality of group means and Levene's test for equality of variances were implemented to test the difference in group means and variances between 3D LS-pfOCE measurements under the normal and CytoD conditions, respectively. The difference was considered significant at $95 \%$ confidence level; the $p$-values are reported in Fig. 3e. For time-lapsed dynamic monitoring of response to cytoD, two-tail Pearson linear correlation was computed between cumulative change in LS-pfOCE measurements and cumulative bead displacements. The correlation was considered significant at $95 \%$ confidence level; the $p$-values are reported on Fig. 3g. All statistical tests were implemented in Python v3.7.7 except the analysis of correlations, which were implemented in MATLAB 2017a.

\section{Reference}


1 Discher, D. E., Janmey, P. \& Wang, Y. L. Tissue cells feel and respond to the stiffness of their substrate. Science 310, 1139-1143 (2005).

2 Eyckmans, J., Boudou, T., Yu, X. \& Chen, C. S. A Hitchhiker's Guide to Mechanobiology. Dev. Cell 21, 35-47 (2011).

3 Doyle, A. D., Carvajal, N., Jin, A., Matsumoto, K. \& Yamada, K. M. Local 3D matrix microenvironment regulates cell migration through spatiotemporal dynamics of contractility-dependent adhesions. Nat. Commun. 6, 8720 (2015).

4 Lampi, M. C. \& Reinhart-King, C. A. Targeting extracellular matrix stiffness to attenuate disease: From molecular mechanisms to clinical trials. Sci. Transl. Med. 10, eaao0475 (2018).

5 Hall, M. S. et al. Fibrous nonlinear elasticity enables positive mechanical feedback between cells and ECMs. Proc. Natl. Acad. Sci. U.S.A. 113, 14043-14048 (2016).

6 Mulligan, J. A., Ling, L., Leartprapun, N., Fischbach, C. \& Adie, S. G. Computational 4D-OCM for label-free imaging of collective cell invasion and force-mediated deformations in collagen. Sci. Rep. 11, 2814 (2021).

7 Pampaloni, F., Reynaud, E. G. \& Stelzer, E. H. K. The third dimension bridges the gap between cell culture and live tissue. Nat. Rev. Mol. Cell Biol. 8, 839 (2007).

8 Legant, W. R. et al. Measurement of mechanical tractions exerted by cells in three-dimensional matrices. Nat. Methods 7, 969-971 (2010).

9 Guilak, F. et al. Control of Stem Cell Fate by Physical Interactions with the Extracellular Matrix. Cell Stem Cell 5, 17-26 (2009).

10 Gilbert, P. M. et al. Substrate elasticity regulates skeletal muscle stem cell self-renewal in culture. Science 329, 1078-1081 (2010).

11 Wong, V. W., Akaishi, S., Longaker, M. T. \& Gurtner, G. C. Pushing Back: Wound Mechanotransduction in Repair and Regeneration. J. Invest. Dermatol. 131, 2186-2196 (2011).

12 Mammoto, T., Mammoto, A. \& Ingber, D. E. Mechanobiology and developmental control. Annu. Rev. Cell Dev. Biol. 29, 27-61 (2013).

13 Paszek, M. J. et al. Tensional homeostasis and the malignant phenotype. Cancer Cell 8, 241-254 (2005).

14 Butcher, D. T., Alliston, T. \& Weaver, V. M. A tense situation: forcing tumor progression. Nat. Rev. Cancer 9 , 108-122 (2009).

15 Levental, K. R. et al. Matrix Crosslinking Forces Tumor Progression by Enhancing Integrin Signaling. Cell 139, 891-906 (2009).

16 Wirtz, D., Konstantopoulos, K. \& Searson, P. C. The physics of cancer: the role of physical interactions and mechanical forces in metastasis. Nat. Rev. Cancer 11, 512-522, doi:10.1038/nrc3080 (2011).

17 Friedl, P. \& Alexander, S. Cancer invasion and the microenvironment: plasticity and reciprocity. Cell 147, 9921009 (2011).

18 Hanahan, D. \& Weinberg, R. A. Hallmarks of Cancer: The Next Generation. Cell 144, 646-674 (2011).

19 Cosgrove, B. D. et al. Rejuvenation of the muscle stem cell population restores strength to injured aged muscles. Nat. Med. 20, 255-264 (2014).

20 Gee, T. W., Richards, J. M., Mahmut, A. \& Butcher, J. T. Valve endothelial-interstitial interactions drive emergent complex calcific lesion formation in vitro. Biomaterials 269, 120669 (2021).

21 Driscoll, K., Cruz, A. D. \& Butcher, J. T. Inflammatory and Biomechanical Drivers of Endothelial-Interstitial Interactions in Calcific Aortic Valve Disease. Circ. Res. 128, 1344-1370 (2021).

$22 \mathrm{Wu}, \mathrm{P}$. H. et al. A comparison of methods to assess cell mechanical properties. Nat. Methods 15, 491-498 (2018).

23 Dufrene, Y. F. et al. Imaging modes of atomic force microscopy for application in molecular and cell biology. Nat. Nanotechnol. 12, 295-307 (2017).

24 Mulligan, J. A., Untracht, G. R., Chandrasekaran, S., Brown, C. N. \& Adie, S. G. Emerging Approaches for HighResolution Imaging of Tissue Biomechanics With Optical Coherence Elastography. IEEE J. Sel. Top. Quantum Electron. 22, 246-265, doi:10.1109/jstqe.2015.2481705 (2016).

25 Larin, K. V. \& Sampson, D. D. Optical coherence elastography - OCT at work in tissue biomechanics. Biomed. Opt. Express 8, 1172-1202 (2017).

26 Kennedy, B. F., Wijesinghe, P. \& Sampson, D. D. The emergence of optical elastography in biomedicine. Nat. Photon. 11, 215-221 (2017).

27 Scarcelli, G. \& Yun, S. H. Confocal Brillouin microscopy for three-dimensional mechanical imaging. Nat. Photon. 2, 39-43 (2008).

28 Remer, I., Shaashoua, R., Shemesh, N., Ben-Zvi, A. \& Bilenca, A. High-sensitivity and high-specificity biomechanical imaging by stimulated Brillouin scattering microscopy. Nat. Methods 17, 913-916 (2020). 
29 Prevedel, R., Diz-Munoz, A., Ruocco, G. \& Antonacci, G. Brillouin microscopy: an emerging tool for mechanobiology. Nat. Methods 16, 969-977 (2019).

30 Wirtz, D. Particle-tracking microrheology of living cells: principles and applications. Annu. Rev. Biophys. 38 , 301-326 (2009).

31 Brau, R. R. et al. Passive and active microrheology with optical tweezers. J. Opt. A: Pure Appl. Opt. 9, S103S112 (2007).

32 Keating, M., Kurup, A., Alvarez-Elizondo, M., Levine, A. J. \& Botvinick, E. Spatial distributions of pericellular stiffness in natural extracellular matrices are dependent on cell-mediated proteolysis and contractility. Acta Biomater. 57, 304-312 (2017).

33 Han, Y. L. et al. Cell contraction induces long-ranged stress stiffening in the extracellular matrix. Proc. Natl. Acad. Sci. U.S.A. 115, 4075-4080 (2018).

34 Juliar, B. A., Keating, M. T., Kong, Y. P., Botvinick, E. L. \& Putnam, A. J. Sprouting angiogenesis induces significant mechanical heterogeneities and ECM stiffening across length scales in fibrin hydrogels. Biomaterials 162, 99-108 (2018).

35 Ashkin, A. Acceleration and trapping of particles by radiation pressure. Phys. Rev. Lett. 24, 156-159 (1970).

36 Leartprapun, N., Iyer, R. R., Untracht, G. R., Mulligan, J. A. \& Adie, S. G. Photonic force optical coherence elastography for three-dimensional mechanical microscopy. Nat. Commun. 9, 2079 (2018).

37 Leartprapun, N., Lin, Y. \& Adie, S. G. Microrheological quantification of viscoelastic properties with photonic force optical coherence elastography. Opt. Express 27, 22615-22630, doi:10.1364/OE.27.022615 (2019).

38 Lin, Y., Leartprapun, N. \& Adie, S. G. Spectroscopic photonic force optical coherence elastography. Opt. Lett. 44, 4897-4900 (2019).

39 Abhilash, A. S., Baker, B. M., Trappmann, B., Chen, C. S. \& Shenoy, V. B. Remodeling of fibrous extracellular matrices by contractile cells: predictions from discrete fiber network simulations. Biophys. J. 107, 1829-1840 (2014).

40 Wang, H., Abhilash, A. S., Chen, C. S., Wells, R. G. \& Shenoy, V. B. Long-range force transmission in fibrous matrices enabled by tension-driven alignment of fibers. Biophys. J. 107, 2592-2603 (2014).

41 Hadi, M. F. \& Barocas, V. H. Microscale Fiber Network Alignment Affects Macroscale Failure Behavior in Simulated Collagen Tissue Analogs. J. Biomech. Eng. 135 (2013).

42 Lai, V. K., Hadi, M. F., Tranquillo, R. T. \& Barocas, V. H. A Multiscale Approach to Modeling the Passive Mechanical Contribution of Cells in Tissues. J. Biomech. Eng. 135 (2013).

43 Zhang, L., Lake, S. P., Barocas, V. H., Shephard, M. S. \& Picu, R. C. Cross-Linked Fiber Network Embedded in Elastic Matrix. Soft Matter 9, 6398-6405 (2013).

44 Mulligan, J. A., Feng, X. \& Adie, S. G. Quantitative reconstruction of time-varying 3D cell forces with traction force optical coherence microscopy. Sci. Rep. 9, 4086 (2019).

45 Planchon, T. A. et al. Rapid three-dimensional isotropic imaging of living cells using Bessel beam plane illumination. Nat. Methods 8, 417-423 (2011).

46 Gao, L. et al. Noninvasive imaging beyond the diffraction limit of 3D dynamics in thickly fluorescent specimens. Cell 151, 1370-1385 (2012).

47 Khaw, I. et al. Flat-field illumination for quantitative fluorescence imaging. Opt. Express 26, 15276-15288 (2018).

48 Choma, M. A., Ellerbee, A. K., Yang, C., Creazzo, T. L. \& Izatt, J. A. Spectral-domain phase microscopy. Opt. Lett. 30, 1162-1164 (2005).

49 Klein, T. \& Huber, R. High-speed OCT light sources and systems [Invited]. Biomed. Opt. Express 8, 828-859 (2017).

$50 \mathrm{Kolb}$, J. P. et al. Live video rate volumetric OCT imaging of the retina with multi-MHz A-scan rates. PLoS One 14, e0213144 (2019).

51 Hall, M. S. et al. Toward single cell traction microscopy within 3D collagen matrices. Exp. Cell Res. 319, 23962408 (2013).

52 Mulligan, J. A., Bordeleau, F., Reinhart-King, C. A. \& Adie, S. G. Measurement of dynamic cell-induced 3D displacement fields in vitro for traction force optical coherence microscopy. Biomed. Opt. Express 8, 1152-1171 (2017).

53 Mark, C. et al. Collective forces of tumor spheroids in three-dimensional biopolymer networks. Elife 9 (2020).

54 Ling, L. et al. Obesity-associated Adipose Stromal Cells Promote Breast Cancer Invasion Through Direct Cell Contact and ECM Remodeling. Adv. Funct. Mater. 30 (2020).

55 Leggett, S. E. et al. Mechanophenotyping of 3D multicellular clusters using displacement arrays of rendered tractions. Proc. Natl. Acad. Sci. U.S.A. 117, 5655-5663 (2020). 
56 Leartprapun, N., Iyer, R. R. \& Adie, S. G. Depth-resolved measurement of optical radiation-pressure forces with optical coherence tomography. Opt. Express 26, 2410-2426, doi:10.1364/OE.26.002410 (2018).

57 Chang, E. W., Kobler, J. B. \& Yun, S. H. Subnanometer optical coherence tomographic vibrography. Opt. Lett. 37, 3678-3680 (2012).

\section{Acknowledgement}

This work made use of the Cornell Center for Materials Research Shared Facilities which are supported through the NSF MRSEC program (DMR-1719875). This work was conducted in part at the Cornell University Biotechnology Resource Center (NIH 1S10RR025502-01) for data collected on the Zeiss LSM 710 laser-scanning confocal fluorescence microscope. This work was funded in part by National Institute of Health (NIBIB-R21EB024747, Adie; NIGMS-R01GM132823, Adie).

\section{Author contributions}

Y.L. and N.L. constructed the LS-pfOCE system. Y.L. conducted all LS-pfOCE measurements. N.L. developed LSpfOCE reconstruction procedure and processed all data. J.C.L. maintained cell culture and prepared collagen matrices and fibrin constructs for live-cell imaging study. Y.L. and N.L. wrote the manuscript. S.G.A supervised the project. All authors helped revise the manuscript.

\section{Competing interests}

The authors declare the following competing interests: N.L. and S.G.A. are listed as inventors on U.S. Patent No. US10072920B2 and US10197379B2. Y.L., N.L., and S.G.A are listed as inventors on U.S. Provisional Patent Application No. 62/968,961. 


\section{Figure captions}

Fig. 1 LS-pfOCE system characterization and validation. a, Schematic of the LS-pfOCE system. Light-sheet radiation-pressure excitation is generated by a cylindrical lens and combined in free-space with the sample arm of a spectral-domain OCT system for interferometric detection of radiation-pressure-induced bead oscillations. See Methods for a full description of the LS-pfOCE system. L: lens, Cyl: cylindrical lens, BS: beam splitter, BSM: beamsteering module, M: mirror, Obj: microscope objective. b, Light-sheet intensity profile measured at the focal plane. c, Measured (red, mean \pm standard deviation from $N=48$ beads) and simulated (blue, Generalized Lorenz-Mie Theory (GLMT), see Supplementary Method 6) lateral light-sheet radiation-pressure profile at its focal plane on 1.9- $\mu \mathrm{m}$ melamine-resin beads. d, Measured axial light-sheet radiation-pressure profile at its lateral center on $1.9-\mu \mathrm{m}$ melamine-resin beads, showing data (blue) and depth-dependent median (black) and median absolute difference (red) from $N=48$ beads. See Methods for light-sheet radiation-pressure force measurement procedure. e, Comparison of $G^{\prime}$ and $G^{\prime \prime}$ in PAAm gels measured by 3D LS-pfOCE (red) and bulk shear rheometry (blue) at $20 \mathrm{~Hz}$. $N=5$ samples for rheometry and $N=225$ and 263 beads for LS-pfOCE in 3T1C and 3T2C gels, respectively. Significant difference in group means is indicated by $* * * p$-value $<0.001$ and n.s. $p$-value $>0.05$.

Fig. 2 Characterization of micromechanical heterogeneity in fibrous collagen matrices. a, Normalized relative variation in stiffness measured by 3D LS-pfOCE in a PAAm gel (3T2C, left) and collagen matrix (C3, right), where larger variation is observed in collagen. $N=181$ and 119 beads for PAAm and collagen, respectively. b, Histograms and statistical distributions of absolute (i.e., without normalization) $G^{\prime}$ and $G^{\prime \prime}$ measurements in a. $\sigma_{\text {FWHM }}$ indicates the FWHM of the distributions. c, En face confocal reflectance microscopy (top, FOV $106 \mu \mathrm{m} \times 106 \mu \mathrm{m}$ ) and OCT images overlaid with color-coded LS-pfOCE measurements of $G^{\prime}$ (middle) and $R$ (bottom) at the focal plane. ROI1 (cyan circles) indicates a stiffer and more solid-like microenvironment in the presence of thick collagen fibres. ROI2 indicates a more compliant and fluid-like microenvironment in the absence of any clear collagen fibres. All OCE images show FOV of $71 \mu \mathrm{m} \times 71 \mu \mathrm{m}$. d, FWHM of the distributions of LS-pfOCE $G^{\prime}$ (red) and $G^{\prime \prime}$ (blue) in PAAm gels and collagen matrices. $N=137,107,105,132$ and 100 beads for P1 (3T1C), P2 (3T2C), C1, C2, and C3, respectively. e, Correlation of LS-pfOCE $G^{\prime}$ (red) and $R$ (blue) to surrogate measures of collagen matrix microarchitectural characteristics. $N=15$ beads in sample C3. Q95, Mean, and Std indicate the 0.95 quantile, mean, and standard deviation of OCT scattering intensity within a $3-\mu \mathrm{m}$ radial distance from the circumference of each bead, respectively (see Methods for further details). CR indicates the Spearman rank correlation coefficient; significance of the correlation is indicated by $* p$-value $<0.05$ and $* * p$-value $<0.01$. Solid lines and shaded regions represent linear fits and $95 \%$ confidence intervals, respectively.

Fig. 3 Live-cell imaging of cell-mediated spatiotemporal variations in ECM micromechanical properties. a, 3D LS-pfOCE measurements of $G^{\prime}$ (color-coded beads) around a cell (white) and $z$-projection of 3D measurements under $\mathbf{b}$, normal condition and $\mathbf{c}, 2$ hours after treatment with CytoD (b and $\mathbf{c}$ show two different cells). Size of the beads is not to scale with the cell body. All images share the same colour scale. (See Supplementary Videos 1 and 2 for visualization of $\mathbf{b}$ and $\mathbf{c}$ from different viewing angles.) d, Histograms and distribution fits of 3D LS-pfOCE measurements in the pericellular (red, $N=205$ beads) and cell-free regions (blue, $N=97$ beads) from 1 cell. $\sigma_{\text {FWHM }}$ indicates the FWHM of the distributions. e, Violin plots of 3D LS-pfOCE measurements around cells under normal (red, $N=1824$ beads) and CytoD (blue, $N=1567$ beads) conditions from 8 cells. The FWHM of each distribution is stated. Significant difference in group means and variance is indicated by $* * p$-value $<0.01$ and $* * * * p$-value $<0.0001$. f, 3D LS-pfOCE measurements of $G^{\prime}$ (left) and $R$ (right) from the data in e as a function of distance to cell under normal (red) and CytoD (blue) conditions. Inset shows power law fits within $\leq 30 \mu \mathrm{m}$ to cell; the decay exponent has a $95 \%$ confidence interval of $-0.19 \pm 0.064$ (red) and $-0.08 \pm 0.024$ (blue). Each data point represents mean \pm standard deviation from $N \geq 20$ beads. $\mathbf{g}-\mathbf{i}$, Boxplots of $G^{\prime}$ (top) and $R$ (bottom) from 5 cells as a function of time after CytoD treatment in regions that experience high-negative (blue, $N=10$ beads), low (grey, $N=16$ beads), and high-positive (red, $N=8$ beads) matrix deformation $S$, respectively. Whiskers represent the full range of values. 


\section{Supplementary Files}

This is a list of supplementary files associated with this preprint. Click to download.

- LSpfOCENatCommfullSupplementaryVideo1submitted.mp4

- LSpfOCENatCommfullSupplementaryVideo2submitted.mp4

- LSpfOCENatCommSupplementary.docx 